\title{
NUMERICAL PREDICTION OF THREE-DIMENSIONAL SHOCK-INDUCED TURBULENT FLOW SEPARATION SURROUNDING BODIES OF REVOLUTION ADJACENT TO A FLAT SURFACE
}

A. D. Grosvenor ${ }^{1}$, A. A. Zheltovodov ${ }^{2}$, and E. K. Derunov ${ }^{2}$

${ }^{1}$ Ramgen Power Systems, LLC. Bellevue WA, USA

${ }^{2}$ Khristianovich Institute of Theoretical and Applied Mechanics Siberian Branch of the Russian Academy of Sciences

Novosibirsk, Russia

\begin{abstract}
Numerical predictions of complex three-dimensional (3D) shock wave/ turbulent boundary layer interactions (SWTBLI) are presented. The configuration studied is a set of two identical cylindrical bodies with conically shaped noses aligned parallel to a Mach 4 stream, adjacent to a flat plate. A series of distances between the bodies are analyzed to test ability of the numerical algorithms employed to capture complex 3D turbulent separation phenomena observed in experiments conducted at the Khristianovich Institute of Theoretical and Applied Mechanics. The numerical scheme employed solves the Reynolds Averaged NavierStokes (RANS) equations, and turbulence closure is provided by the low Reynolds number Spalart-Allmaras one-equation model. Among other areas of concentration discussed herein are the properties of the computational grid required to capture the flow physics sufficiently to reproduce the complex boundary layer separation patterns. The value of point-enrichment type adaptation over block-enrichment is demonstrated, and the shock capturing properties of 2nd order central and upwind discretization schemes are compared.
\end{abstract}

\section{INTRODUCTION}

The predictions discussed herein represent experiments [1-3] studying the aerodynamic interference of two conically shaped cylindrical bodies of varying proximity aligned parallel to a Mach 4 stream, adjacent to a flat plate (Fig. 1a).

This is an Open Access article distributed under the terms of the Creative Commons Attribution-Noncommercial License 3.0, which permits unrestricted use, distribution, and reproduction in any noncommercial medium, provided the original work is properly cited. 


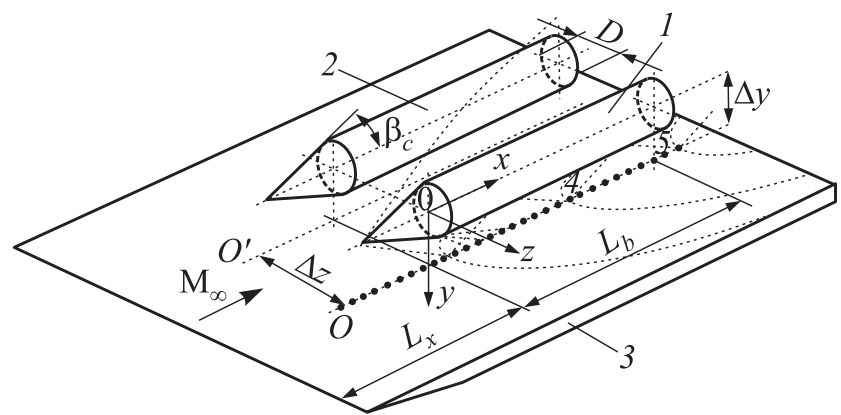

(a)

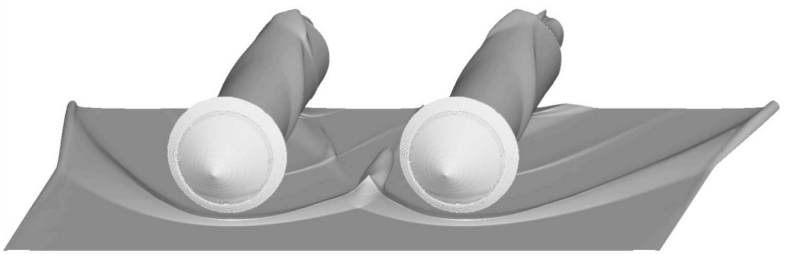

(b)

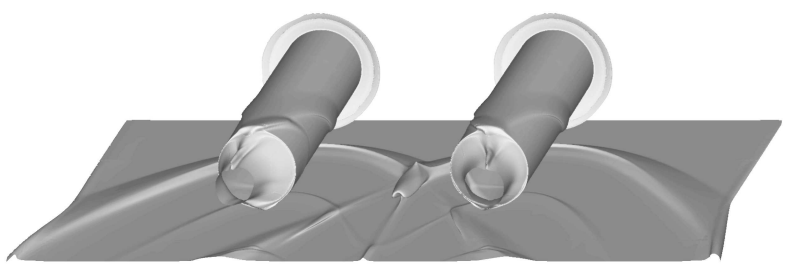

(c)

Figure 1 (a) Schematic of experiment (1 - first body; 2 - second body; and 3 flat plate); (b) forward looking downstream; and (c) aft looking upstream - views of surfaces of constant velocity demonstrating 3D separating flow structures from bodies and plate at $\Delta z / D=3$

As was demonstrated in the mentioned papers, crossing shock waves generated around the bodies, and their interaction with the turbulent boundary layers adjacent to surfaces of the flat plate and bodies initiates the emergence of complex 3D separated flow structures. Figures $1 b$ and $1 c$ indicate the regions of flow separation from the bodies and plate from one of the computations run for this study at $\Delta z / D=3$. Surfaces of constant velocity are shown here to provide an overview of the $3 \mathrm{D}$ flowfield, where one can observe the forebody bow shock waves and their subsequent impingement on the plate and reflections with each other and the solid surfaces. The resulting raised areas of these surfaces 
indicate the displacement effect of 3D separation zones, which will be shown in greater detail below in terms of limiting surface streamlines. Topology of these separated flows as well as the shock structures, pressure distributions, and aerodynamic forces depend on the distance between these bodies. The complex flowfield cannot be predicted based on the assumption of inviscid flow imposed by the solution of the Euler equations, but this simplified approach was employed for preliminary prediction of inviscid shock waves structure, surface pressure distributions, and the bodies' aerodynamic forces and moment coefficients [3]. The present work was directed at validation of a RANS approach to predicting the complex 3D shock wave/boundary layer interaction physics of this flow. The Jameson-Schmidt-Turkel [4] type 2nd order finite volume scheme was primarily employed, and turbulence was closed using the Spalart-Allmaras one-equation model [5]. The low Reynolds number version of this model was applied, meaning that integration was conducted through the boundary layers down to the viscous sublayer.

\section{EXPERIMENTAL TEST CONDITIONS}

The supersonic tunnel conditions were set to produce Mach 4 freestream dried air flow at the unit Reynolds number of approximately $55 \cdot 10^{6} \mathrm{~m}^{-1}$. Inflow total pressure and temperature were $1.074 \cdot 10^{6} \mathrm{~Pa}$ and $291 \mathrm{~K}$, respectively. The adiabatic wall condition was realized in the experiment and the plate turbulent boundary layer thickness was 1.8-2 mm immediately upstream of the impingement location of the generated bow shock waves on the plate. The Reynolds numbers in terms of boundary layer thickness $\operatorname{Re}_{\delta}=(0.7-1.23) \cdot 10^{5}$ corresponded to its turbulent state immediately after the termination of laminar-turbulent transition. The boundary layer on the bodies was considered as turbulent, although the laminar-turbulent effects must in reality be considered, which would be appropriate in the next stage of research. The body diameter was $50 \mathrm{~mm}$, the nose cone included angle ranged from $10^{\circ}$ to $30^{\circ}\left(30^{\circ}\right.$ is the focus herein - forebody angle $=60^{\circ}$ ) and the body length measured from the nose cone base was $250 \mathrm{~mm}\left(L_{b} / D=5\right)$. The bodies were set at a vertical distance of $48 \mathrm{~mm}$ from the plate to their centerline axes $(y / D=0.96)$. The representation depicted in the figures and modeled in the computational grid is simplified, as the bodies were mounted on base-stings connected to balances. Disturbances induced by the stings existed downstream of the regions concentrated on in this study. Distances between the bodies tested ranged from $\Delta z / D=1.06$ to 3 . Four basic test configurations were analyzed in the present study with the values of interbody distances $\Delta z / D=3,1.8,1.4$, and 1.06 , respectively. In the experimental work, several additional configurations were measured, but four were chosen for this study over the range of $\Delta z / D$ values tested. Measurements taken for comparison included static pressure taken with a dense set of static taps over the 
plate surface, Schlieren photography of the shock system induced by the bodies, oil flow visualization using a transformer oil-gaseous soot mixture on the plate and body surfaces as well as balance measurements of aerodynamic forces and moments on one of the bodies. The plate was supplied with a drained insert and 100 drainage orifices of diameter $0.5 \mathrm{~mm}$ at increments of $3.5 \mathrm{~mm}$ along a single line directed along the flow. To obtain the panorama of pressure distributions in the region with dimensions $346.5 \times 308 \mathrm{~mm}$ (on the basis of measurements at 8900 points), it was shifted in the horizontal direction with respect to bodies at a distance of up to $77 \mathrm{~mm}$ at discrete steps of $3.5 \mathrm{~mm}$ with the aid of a special pneumatic device [1].

\section{NUMERICAL SCHEME}

Two codes were employed in this study: FINE/Turbo and FINE/Hexa of Numeca (referred to hereafter as $\mathrm{F} / \mathrm{T}$ and $\mathrm{F} / \mathrm{H}$, respectively). Both solve the compressible form of the RANS equations, and the Spalart-Allmaras one-equation turbulence closure [5] was chosen. $\mathrm{F} / \mathrm{T}$ and $\mathrm{F} / \mathrm{H}$ employ a cell-centred finitevolume density based scheme modeled after the 4-stage Runge-Kutta time integration method of Jameson, Schmidt and Turkel [4] — commonly referred to as JST. While F/T conducts solutions on structured multiblock grids, F/H employs an unstructured hexahedral grid generated via octree-refinement. The agglomeration-type multigrid convergence acceleration is employed in both codes (in $\mathrm{F} / \mathrm{T}, 8$ adjacent cells are agglomerated to one preserving the structured grid index system, and in $\mathrm{F} / \mathrm{H}$ agglomeration is unstructured). $\mathrm{F} / \mathrm{H}$ also offers solution-adaptive grid refinement. Further specifics of the F/T solver algorithm were supplied by Hakimi [6], previous computational fluid dynamics (CFD) validation of $\mathrm{F} / \mathrm{T}$ specific to SWBLI was given by Grosvenor [7] and details of $\mathrm{F} / \mathrm{H}$ were provided by Patel [8] and Delanaye et al. [9].

\section{NUMERICAL MODEL}

Figure 2 displays views of both the structured multiblock and adapted unstructured computational grids employed for the $\Delta z / D=3$ test configuration. Inflow velocity and static pressure and temperature were set to reproduce the experimental conditions of Mach 4 flow at a total pressure and temperature of $1.074 \cdot 10^{6} \mathrm{~Pa}$ and $291 \mathrm{~K}$, respectively. Half of the physical domain was modelled by employing a zero-gradient Neumann boundary condition at the centreline surface. The opposite surface in the $z$ direction was extended to the tunnel wall location using a coarser grid resolution. A slip boundary condition was set between the plate edge and tunnel wall, and the body and plate surfaces were set 


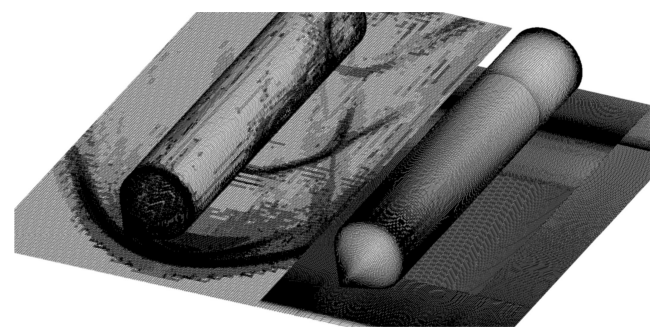

(a)
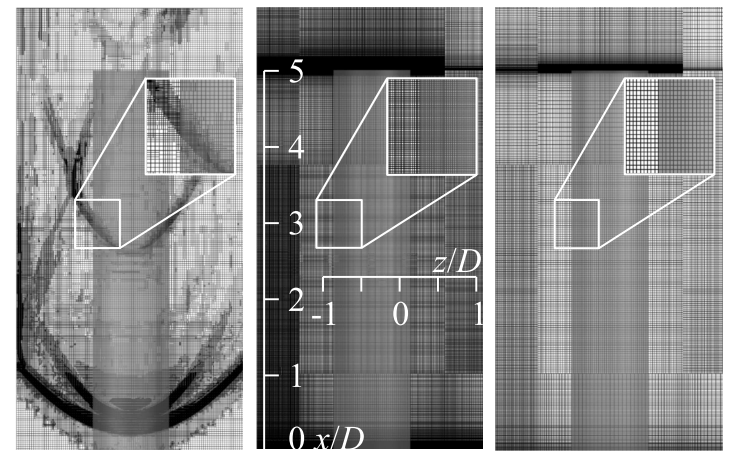

(b)

Figure 2 Views of structured and unstructured computational grids: $(a)$ 3D view of structured multiblock grid (right) and solution-adapted hexahedral unstructured grid (left); and (b) plate computational grids: left - adapted unstructured 25 million cell, center — structured 56 million cell, right — structured 7 million cell

to no-slip. The remaining boundaries were set to supersonic outflow conditions where flow is assumed to be supersonic and extrapolated from the interior of the domain. Coarser versions of the structured grid were run, and sub-blocks were constructed and individually refined to reach a reasonably grid-independent solution (i. e., block-enrichment was conducted to maximize resolution of the relevant phenomena) - judged in terms of shock structures and limiting surface streamline patterns on the flat plate. Grid refinement was particularly employed in the blocks between the body and plate, and at the body's base - while coarser resolution was set above the body. Simultaneously, the first grid nodes from the plate and body solid surfaces were set such that average $y_{1}^{+}$values did not exceed unity. The total number of cells in this final grid was 56 million which might be considered a very high number for such a seemingly simple geometric configuration. For comparison, two coarser structured grids were run at 7 and 1 million grid cells. Details of the two finer structured grids are listed in Table 1, where \# b.l. indicates number of cells in the plate boundary layer 
Table 1 Structured grid details

\begin{tabular}{cccc}
\hline \# total cells & $y_{1}^{+}$ & \# b.l. & \# cells across shocks \\
\hline $56 \cdot 10^{6}$ & $0.15-1.2$ & 40 & $3-6$ \\
$7 \cdot 10^{6}$ & $0.25-2.5$ & 20 & $1-3$ \\
\hline
\end{tabular}

just upstream of the point of bow shock impingement. Computations were also performed using $\mathrm{F} / \mathrm{H}$ employing solution-adaptive grid refinement, which lead to a grid resolution consisting of 25 million cells resolved to the $3 \mathrm{D}$ shock wave structures. As might be expected, solution-adaptive point-enrichment type grid refinement optimizes the placement of grids cells to reduce the overall required grid size to adequately capture the highly 3D concentrated SWBLI phenomena.

\section{DISCUSSION OF PREDICTIONS AND COMPARISON OF MEASUREMENTS WITH COMPUTATIONAL FLUID DYNAMICS}

Predictions of $\mathrm{F} / \mathrm{T}$ and $\mathrm{F} / \mathrm{H}$ follow, primarily employing the 2nd order central difference spatial discretisation JST scheme, and the Spalart-Allmaras oneequation turbulence closure. The 2nd order Roe Flux-Difference Splitting (FDS) upwind scheme [10] and Symmetric Total Variation Diminishing (STVD) scheme of Yee [11] in conjunction with the van Leer and superbee flux limiters were also selectively tested, and these results will be discussed additionally in the next section. Figure $3 a$ displays a Schlieren photograph indicating the shock wave structure over a vertical plane at the body centerline (note EW signifies expansion wave). In Fig. $3 b$, the predicted streamwise density gradient for the same configuration is provided for comparison. One can observe that the results compare well with experiment in terms of shock angles and presence of both separation shock wave $\left(1_{S}\right)$ and its continuation $\left(2^{\prime}\right)$ above the conical bow shock (1), and terminal shock $\left(1_{R}\right)$ from the reattachment region. Upon merging, shocks $2^{\prime}$ and $1_{R}$ form a single shock wave 2 , followed by the next downstream reflected shock (3). Figures $3 c$ and $3 d$ display the shock wave structure produced by the bodies spaced at $\Delta z / D=3$ in terms of predicted Mach number contours. Over the $z$-plane (see Fig. 3c), one can observe the shock structure induced by the forebody and arising separation zone in the vicinity of the bow shock interaction with the boundary layer on the plate surface as well as subsequent shock reflections between the body and plate. As seen from the calculations, additional downstream reflected shocks $(4,5)$ appear between the body and plate downstream of shock 3 (indicated in Fig. $1 a$ and shown in Figs. $3 c$ and $4 a$ ). The 


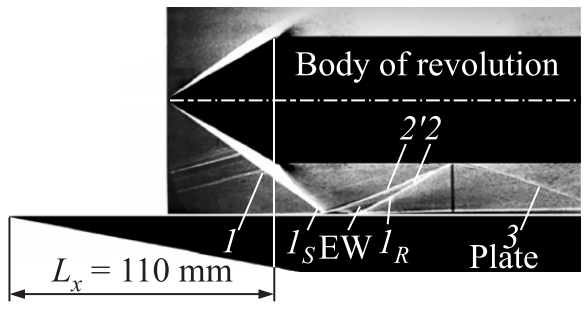

(a)

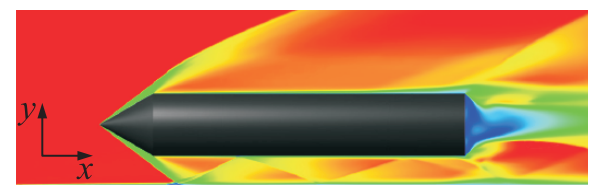

(c)

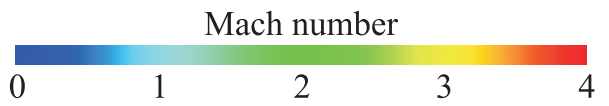

Stream wise density gradient $/ \mathrm{kg} / \mathrm{m}^{3}$

$\begin{array}{llll}-100 & -60 & -20 & 20\end{array}$

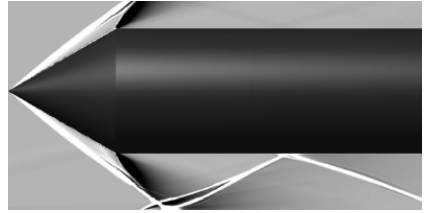

(b)

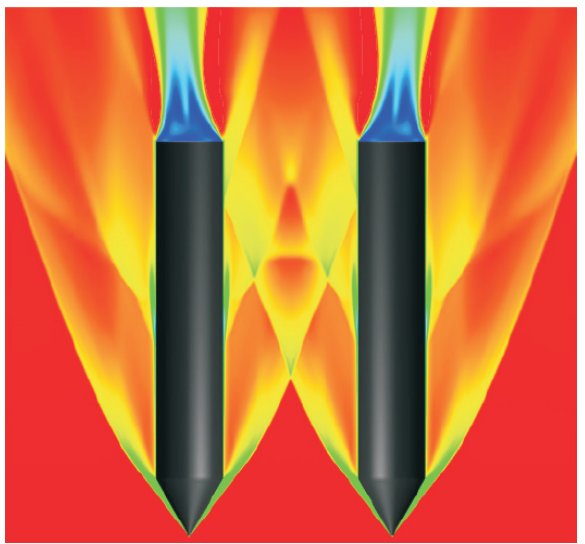

$(d)$

Figure 3 Views of flow field at $\Delta z / D=3$ : (a) Schlieren photograph taken over vertical plane ( $z$-plane) at body centerline; $(b)$ predicted shock wave structure displayed in terms of streamwise density gradient; $(c)$ predicted Mach number contours over $z$-plane; and $(d)$ a plane extending in cross-stream direction ( $y$-plane) along body centerline. (Refer Grosvenor et al., p. 125.)

$y$-plane view (see Fig. $3 d$ ) shows the shock structure between the bodies and the base separation zones downstream of them.

The evolution of complex crossing shock patterns for the four configurations considered in this study with varying interbody distance decreasing from $\Delta z / D=3$ to 1.06 is demonstrated in Figs. 4, $5 a, 5 e$, and $5 h$ in accordance with the CFD predictions. The conical bow shocks induced by the forebodies are shown to merge and reflect in a complex $3 \mathrm{D}$ manner between the bodies and on the plate. Their structure differs markedly from the earlier predictions [3] that employed the Euler equations, due to the emergence of 3D secondary flows and separation zones in the boundary layer on the plate and bodies' surfaces described in detail earlier in accordance with the experiments $[2,3]$. Emerging separation 

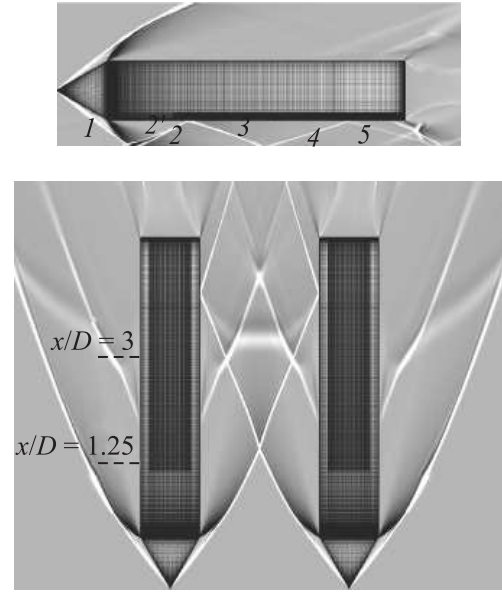

(a)
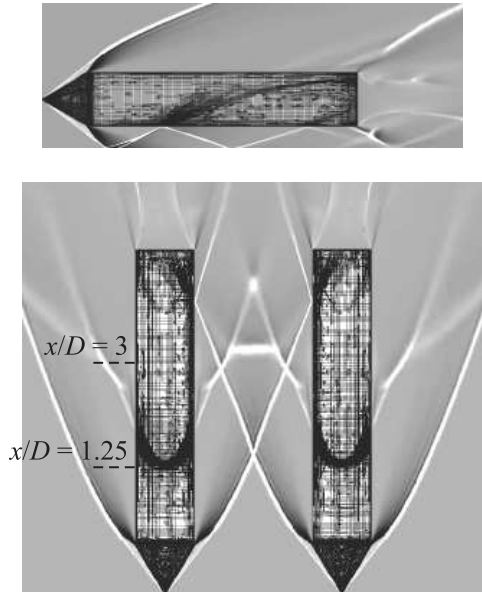

(b)

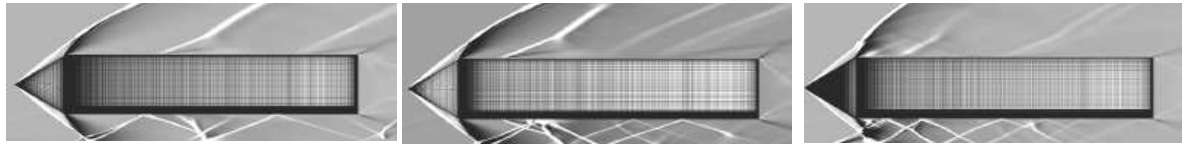

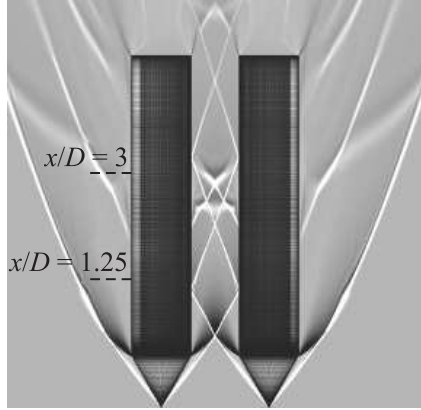

(c)

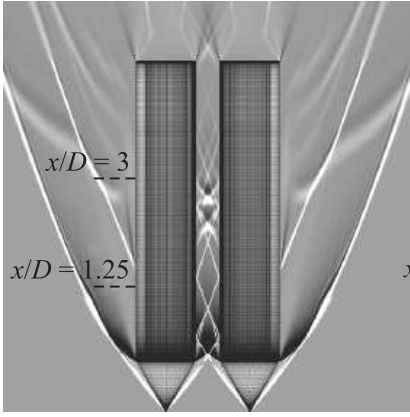

(d)

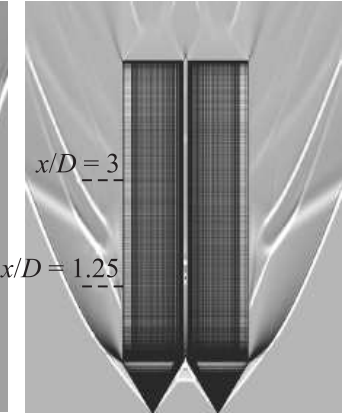

(e)

Figure 4 Cutting planes in three coordinates displaying predicted streamwise density gradient contours for $x$-plane and $y$-plane views taken at body centerline: $(a) \Delta z / D$ $=3.0$, structured 56 million cell; $(b) \Delta x / D=3.0$, unstructured adapted 25 million cell; structured 56 million cell; and $(c) \Delta z / D=1.8,(d) \Delta z / D=1.4$, and $(e) \Delta z / D=1.06$

and attachment zones and their interaction with a basic 'inviscid' shock wave structure change the flowfield significantly (further discussion of Fig. $5 b-5 d, 5 f$, $5 g, 5 i$, and $5 j$ is given in the next section in the context of comparing shock capturing strategies). As seen in Fig. 6, the computations predict different stages of crossing bow shock interaction in the external flow between the bodies with 


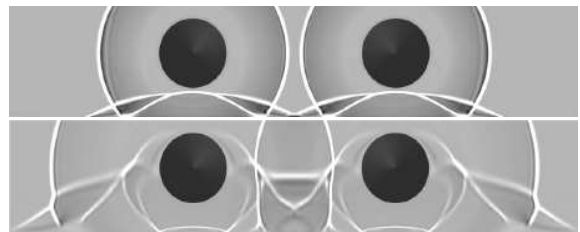

(a)

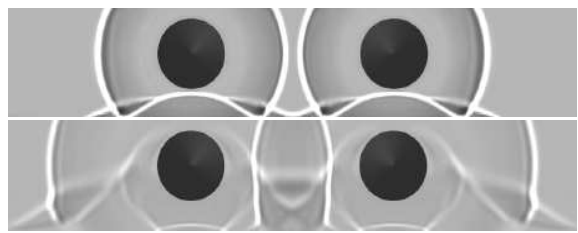

(b)

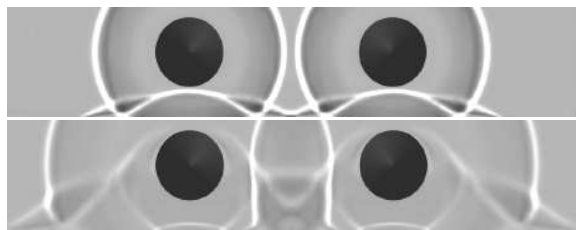

(c)

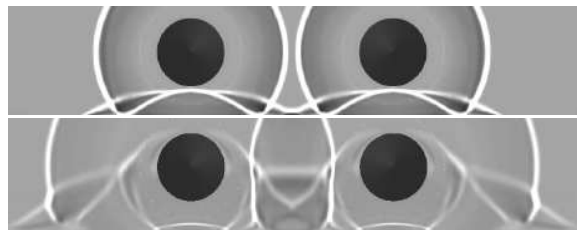

(d)

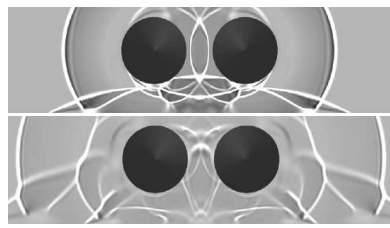

(e)

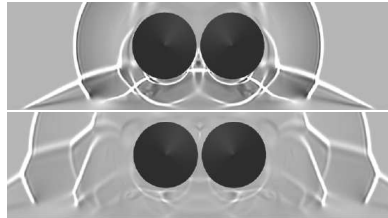

(h)

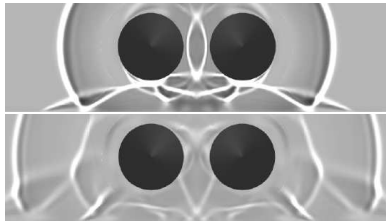

(f)

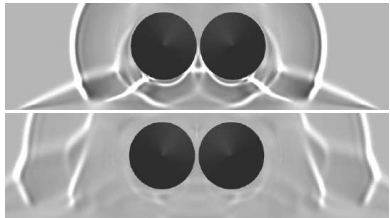

(i)

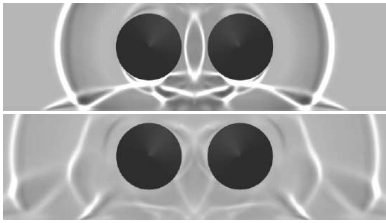

(g)

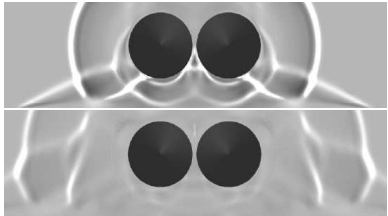

(j)

Figure 5 Cross-sectional views of shock system indicated by streamwise density gradient contours at $x / D=1.25$ (upper figures), and $x / D=3$ (lower figures): $(a)$ (d) $\Delta z / D=3 ;(e)-(g) 1.4 ;(h)-(j) \Delta z / D=1.06 ;(c),(g)$, and $(j)$ 2nd order upwind STVD-van Leer; $(d)$ 2nd order upwind Roe-superbee; remaining $(a),(b),(e),(f),(h)$, and $(i) \mathrm{JST} ;(a),(e)$, and $(h) 56 \cdot 10^{6}$ cells; and $(b)-(d),(f),(g),(i)$, and $(j) 7 \cdot 10^{6}$ cells

decreasing distance $\Delta z / D$. The regular crossing shock structure at $\Delta z / D=1.8$ is followed by the appearance of a small Mach stem as the interbody distance is reduced to $\Delta z / D=1.4$, and a distinct Mach stem is observed at $\Delta z / D=1.06$. This trend and the specific structures are predicted well in comparison with experiment.

One can observe a progressional shift in the complex 3D viscous/inviscid interaction of the shock waves and turbulent boundary layers as the distance between bodies, $\Delta z / D$, decreases (see Figs. 4 and 5). Strong adverse pres- 


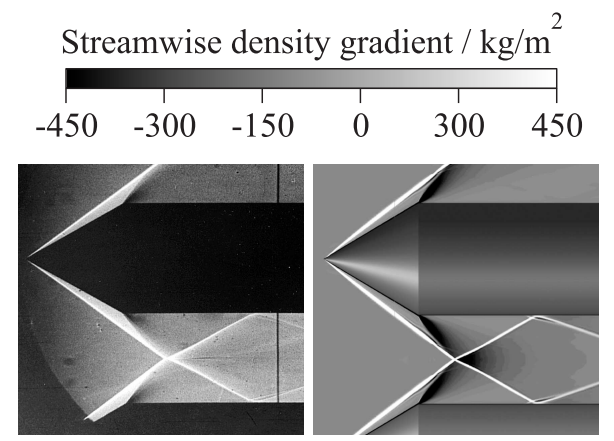

(a)

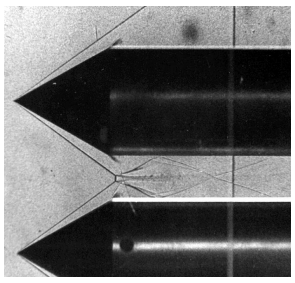

(b)

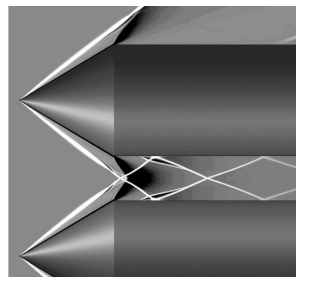

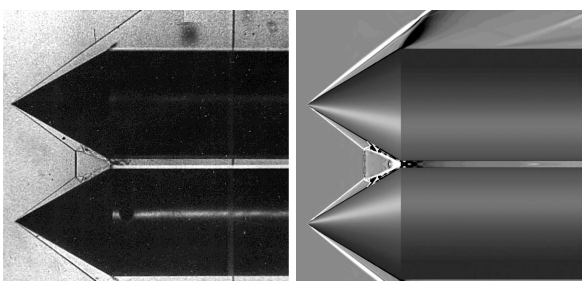

(c)

Figure 6 Comparison of experimental Schlieren photograph (left figures) with predicted crossing shock wave structure (right figures) between the bodies: (a) regular interaction of crossing bow shocks $(\Delta z / D=1.8) ;(b)$ incipience of small Mach stem $(\Delta z / D=1.4)$; and $(c)$ flow stage with distinct Mach stem $(\Delta z / D=1.06)$. Note: legend applies to CFD figures

sure gradient regions are subsequently induced at the plate surface, as shown in Fig. 7. The experimental measurements are depicted on the left side of the figure (decreasing $\Delta z / D$ ), showing the two main pressure maximums ( $A$ and $B$ ) which correspond to attachment nodes $\left(N_{1}\right.$ and $\left.N_{1}^{\prime}\right)$ under the bodies depicted in the limiting surface streamline plots in Fig. 8 (left side of figure, decreasing $\Delta z / D)$. The central peak $(C)$ appears at the node point $\left(N_{0}\right)$ in developing the central separation zone between the bodies, as described further in [2]. The experimentally indicated topology of surface streamlines with various marked singular points has been traced from oil flow visualization and convergence (or separation) and divergence (or reattachment) lines are labelled as $S$ and $R$, respectively. The predicted limiting streamlines from CFD have been generated by computing velocity vector stream-traces projected to the planar cuts taken at the location of the first layer of grid nodes off the body and plate surfaces. Predicted static pressure coefficient distributions on the flat plate for each of the four configurations (see Fig. 7, right) are compared with measurements in terms of pressure coefficient surfaces. The structured grid CFD prediction is seen to 

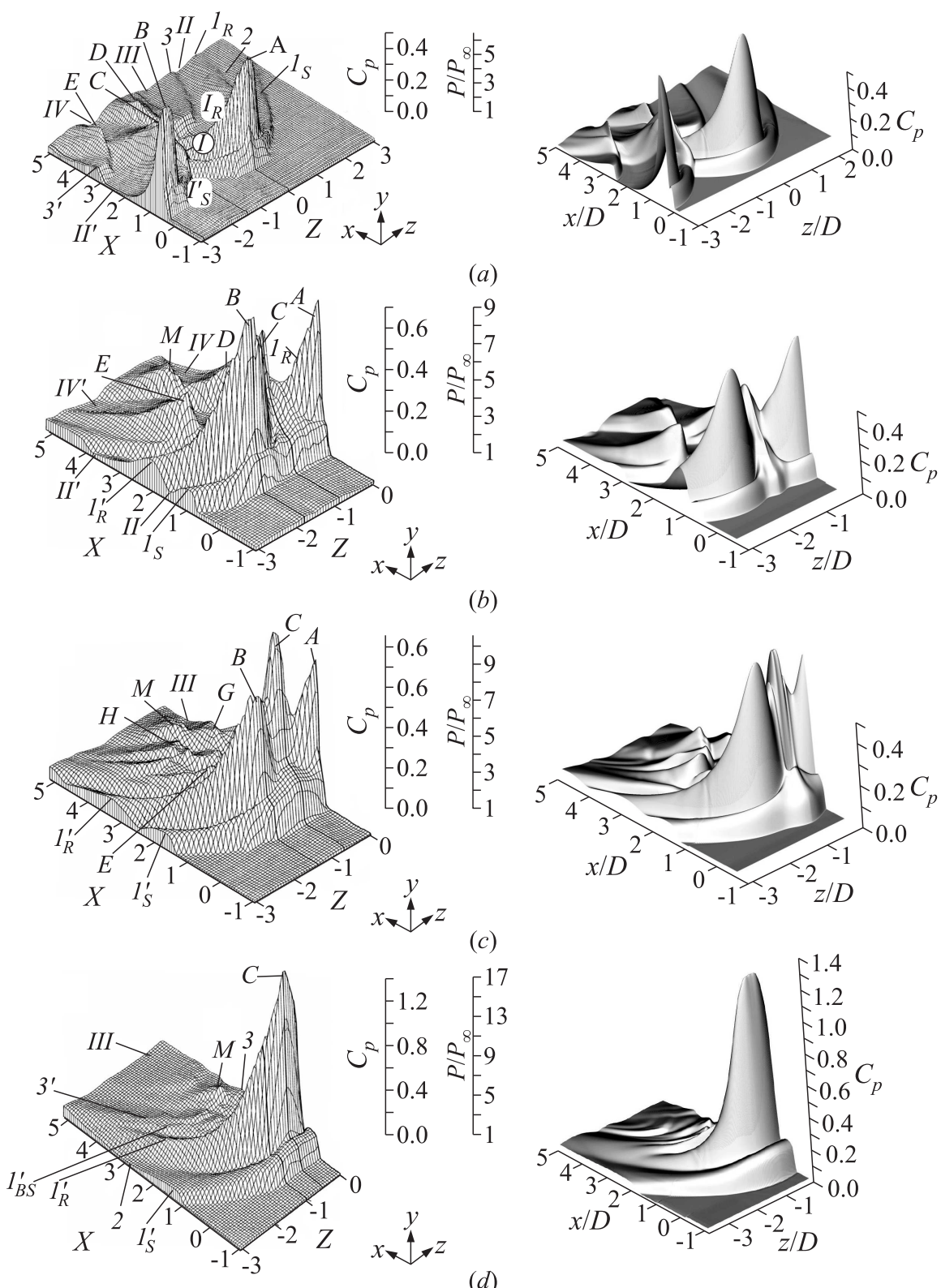

(d)

Figure 7 Axonometric views of plate static pressure coefficient surfaces comparing experimental measurements (left) with predictions (right): (a) $\Delta z / D=3.0$; (b) 1.8; (c) 1.4 ; and $(d) \Delta z / D=1.06$ 

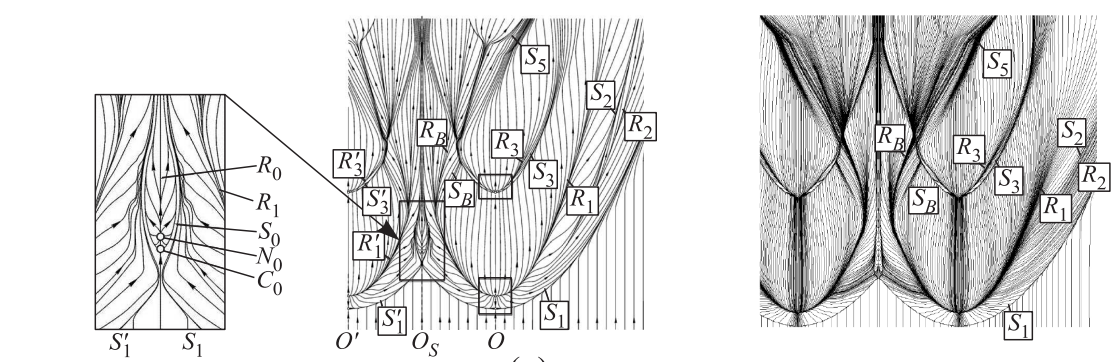

(a)
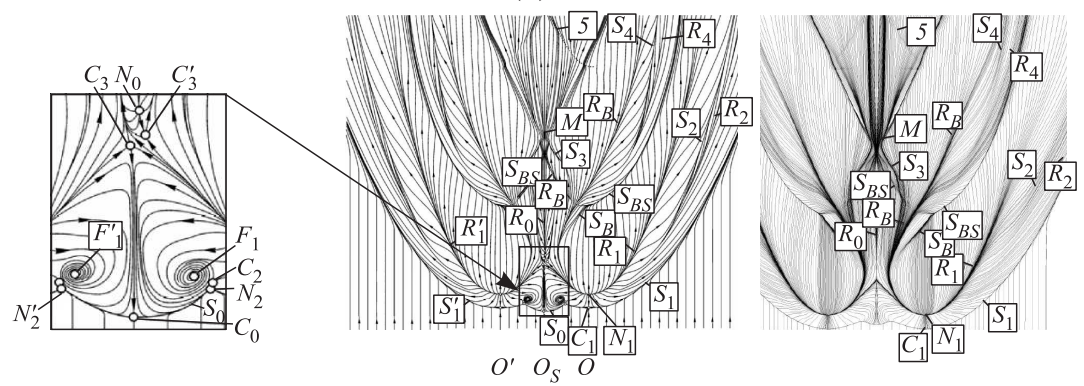

(b)
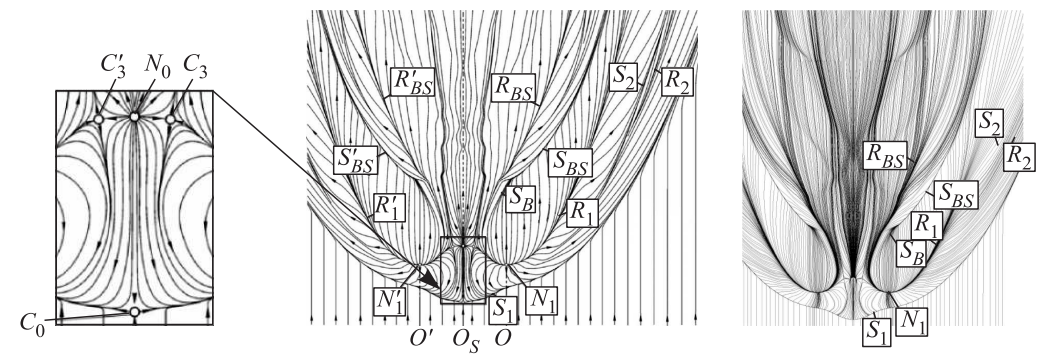

(c)
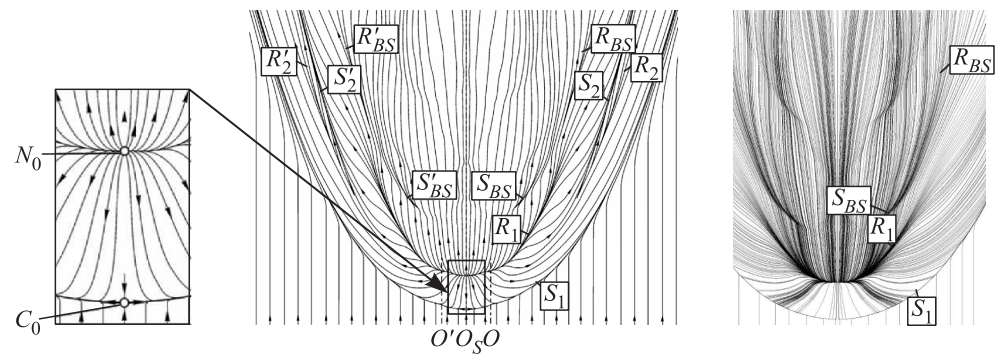

(d)

Figure 8 Predicted plate surface streamlines compared to experimental oil flow visualization 


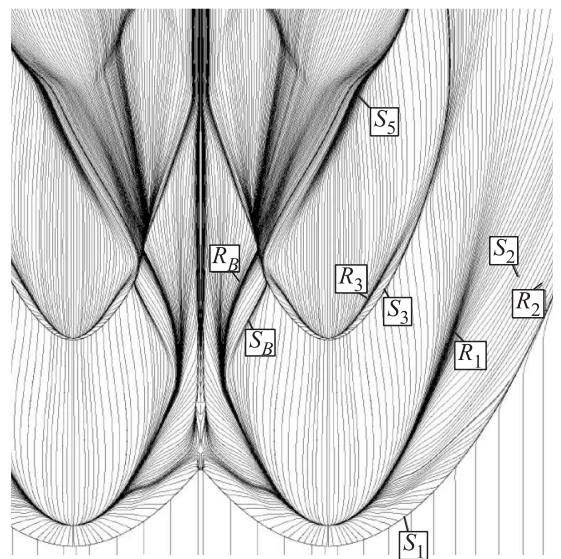

(a)

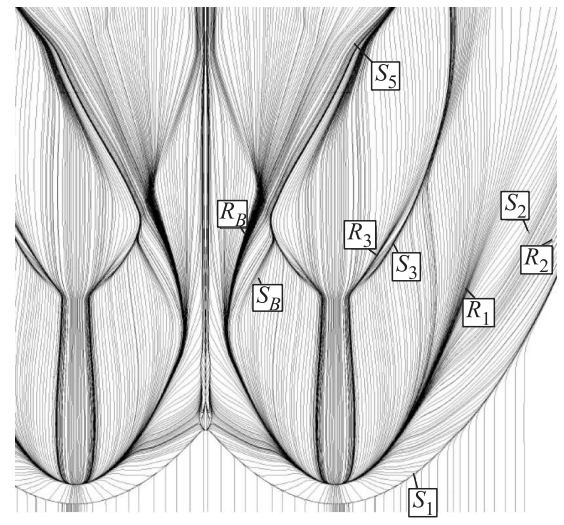

(c)

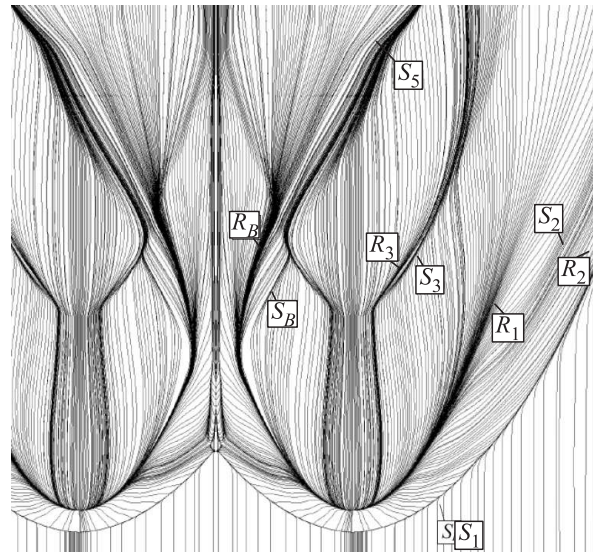

(b)

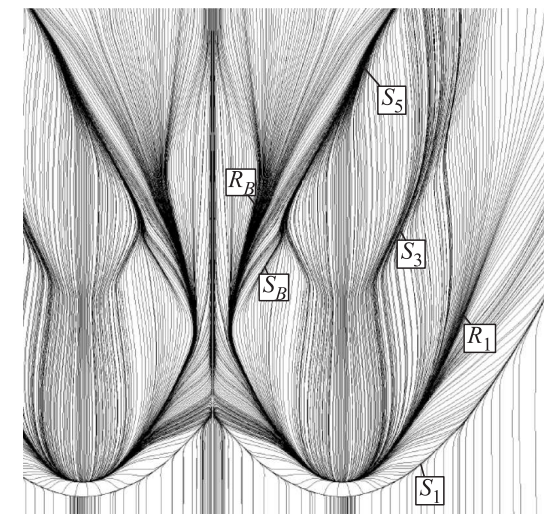

(d)

Figure 9 Predicted plate surface streamlines for $\Delta z / D=3$ predicted with different computational grid topologies and resolutions: $(a)$ adapted unstructured; 25 million grid cell; (b) 7 million grid cell, 2nd order upwind (STVD); (c) 7 million grid cell; and (d) 1 million grid cell

reproduce the pressure field quite well in terms of magnitudes and trends. Respectively, the computed surface flow patterns (Figs. 8 and 9) tend to follow experiment well. For instance, separation lines $S_{1}, S_{3}$, and $S_{5}$ on the plate at $\Delta z / D=3.0$ (see Figs. $8 a$ and 9 ) and their associated reattachment lines are shown to compare well with experiment. These three separation lines can be seen to occur consistently with the first bow shock, and reflected second and third shock waves directed from the body to the plate intersecting the plate 
surface from left to right (see Figs. $3 a$ and $3 b$ ). Separation line $S_{B}$ is also well captured. It is perhaps one of the most complex separation locations, as it is due to essentially $3 \mathrm{D}$ interaction of crossing shock waves separating the plate boundary layer. One perhaps more complex location is the convergence region $S_{5}$ in the vicinity of the base flow, and this is also seen to be captured quite well. Conversely, secondary convergence line $S_{2}$ and divergence line $R_{2}$ are shown to be underpredicted in terms of definition and streamline angle. The underprediction here is largely due to the fact that grid resolution has been concentrated in the zones between bodies, and lower resolution has been chosen on the outside. Note that one can see a better resolved set of separation and reattachment lines $S_{3}$ and $R_{3}$ from the unstructured solution-adapted grid computation in Fig. $9 a$. This zone exists in the region of $x / D=3$ where it is shown in Fig. 2 that the adapted grid employs the highest local resolution. One can observe a similar prediction accuracy demonstrated for each of the four configurations, and the impact of coarsening the grid is depicted in Fig. 9 for $\Delta z / D=3.0$ which displays an expected trend of reducing definition as the grid is coarsened due to artificial diffusion.

Note that from the experimentally indicated streamlines, there appears to be some potential for flow asymmetry at $\Delta z / D=1.8$ (see Fig. $8 b$ ). This may offer at least part of the explanation for the lack of focus nodes predicted for this case. One can see the predicted streamlines curling up in this region, but focus nodes are not present. In the experiment, they seem to be not completely symmetric in size. The assumption of symmetry imposed on the computations obviously prevents the prediction of such phenomena.

Another potential reason for disagreement is that the turbulence closure employed is predicting higher levels of turbulence locally than what is realistic. The asymmetry could potentially arise as a time-varying phenomena, which would further explain differences in prediction. An alternative explanation could be some geometric asymmetry of the test model caused by small deformations [2] which would not have been included in the numerical model. In general, the separated flow patterns over both the bodies and plate for all cases are reproduced well in the predictions, and the impact of coarser grid resolution (e.g., the 1 and 7 million cell grids in Fig. 9) can be seen to be a loss of resolution of the key separating zones at $\Delta z / D=3.0$. In accordance with Figs. 7 and 8 , the cardinal reconstruction of separated flow on plate surface occurs with decreasing distance between the bodies to the minimal value $\Delta z / D=1.06$ at which forming the Mach stem (see Figs. $4 e$ and $6 c$ ) indicates an 'unstart' phenomenon in the limited space between the bodies and plate.

Predicted static pressure coefficient distributions on the flat plate are compared quantitatively with experimental data for $\Delta z / D=3.0$ and 1.4 in Fig. 10. The structured grid CFD simulation is seen to reproduce the pressure field quite well in terms of magnitudes and trends. The largest differences appear to be in the vicinity of reflected shock 3 , where the pressure coefficient predictions show 


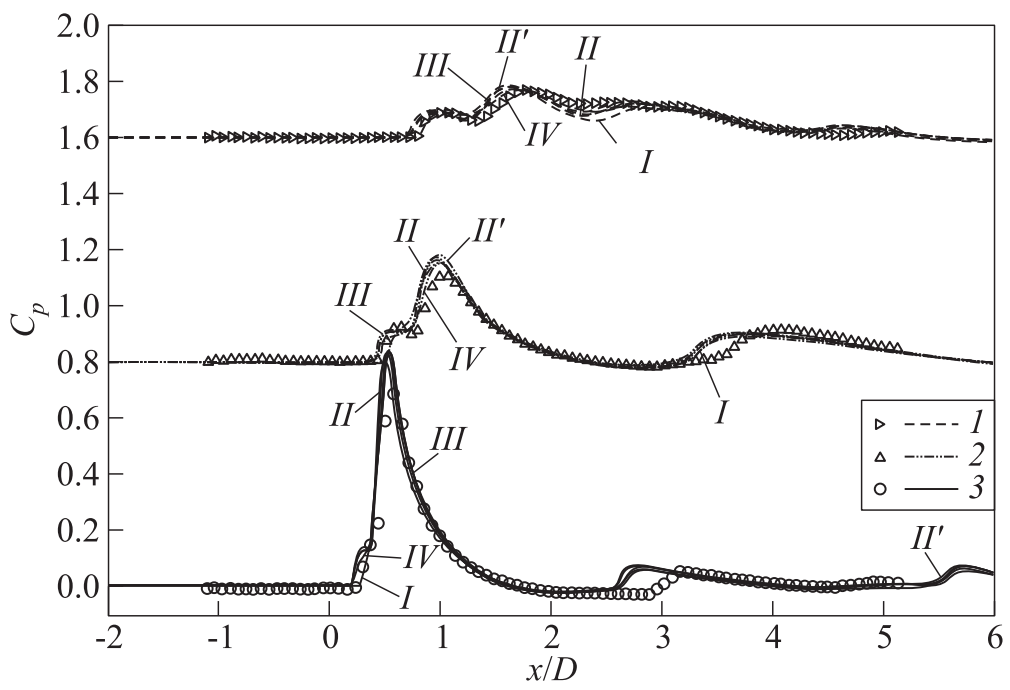

(a)

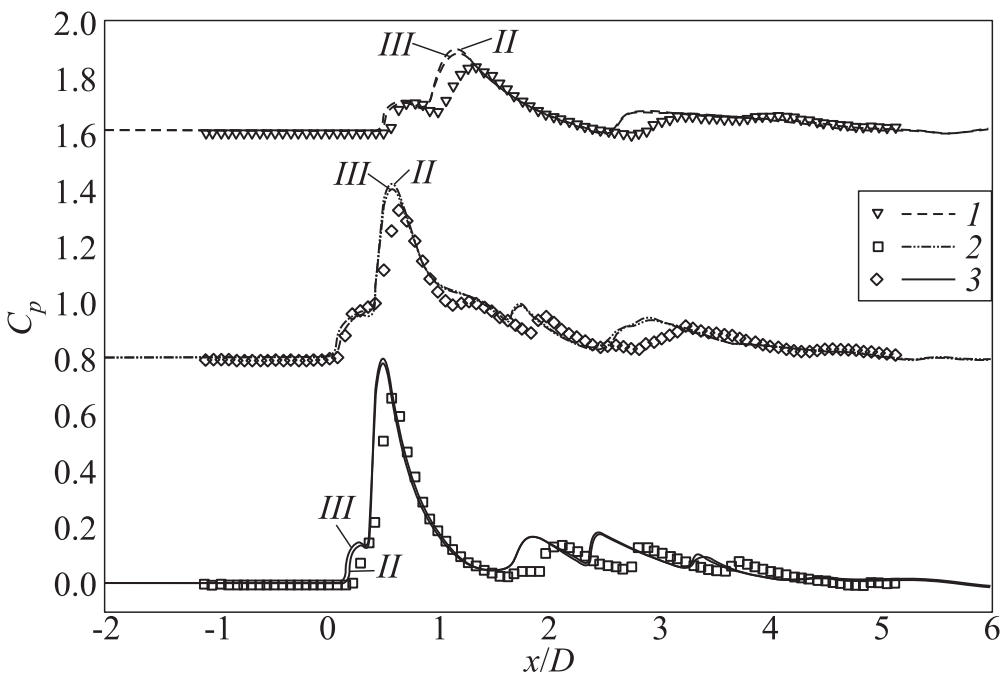

(b)

Figure 10 Predicted (curves) plate static pressure coefficients compared to experimental measurements [2] (symbols): ( a) $\Delta z / D=3$; (b) $\Delta z / D=1.4: 1$ million grid cell $(I) ; 7$ million grid cell $(I I)$, 2nd order upwind $\left(I I^{\prime}\right) ; 56$ million grid cell $(I I I)$ structured grids; 25 million cell adapted unstructured grid $(I V) ; 1-z / D=-1.12$ from body centerline; $2--0.7$; and $3-z / D=0.0$ from body centerline 


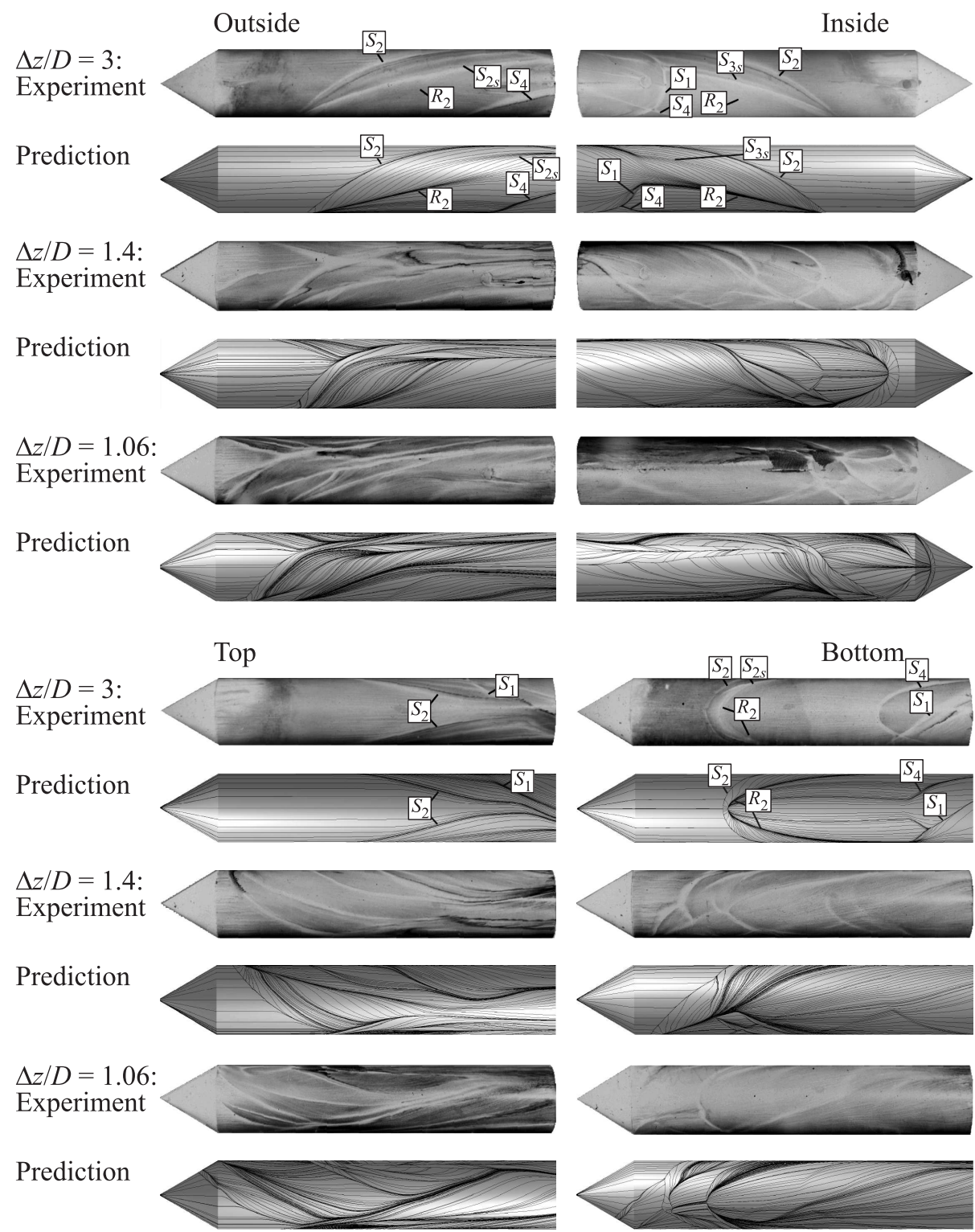

Figure 11 Comparison of predicted $\left(56 \cdot 10^{6}\right.$ cells $)$ limiting surface streamlines with surface oil flow visualization on body 1 
a subsequent pressure rise slightly upstream of the true position. This is the previously mentioned region surrounding $x / D=3$, and one can conclude from the comparison of finest structured grid and unstructured adapted grid that the difference between prediction and experiment is not due to grid resolution. It is expected that this is a region where the impact of different turbulence closure options would be of most interest. In addition to the highly $3 \mathrm{D}$ nonequilibrium state of turbulence expected to be present here, the expansion downstream of the forebody may also be accelerating the flow such that local relaminarization and subsequent transition occurs. The impact of higher levels of turbulence closure sophistication is being tested in the next phase of study.

As seen in Figs. 4 and 5, a complex system of shock waves forming around the bodies interact also with the boundary layer on their surfaces. The computed surface flow pattern for the configurations shown tends to follow experiment well (Fig. 11). For instance, at $\Delta z / D=3.0$, the number of the separation and attachment lines $S_{2}, R_{2}, S_{4}$, and $R_{4}$ corresponds to the influence of reflected shocks 2 and 4 from the plate to body (see additionally Figs. $3 a$ and $3 c$ ), which penetrate and diffract around the bodies. The separation line $S_{1}$ arises from the conical bow shock wave penetrating from the second body to the surface of the first. Lines $S_{2 s}$ and $S_{3 s}$ indicate secondary separations. In accordance with experiment and computations, decreasing $\Delta z / D$ leads to a significant rise of separation zones on the body surface and in conditions of the 'unstart' phenomenon at $\Delta z / D=1.06$, they penetrate the surfaces of the conical forebodies. Note that local grid resolution between the forebodies was increased in this specific configuration to better capture the unstarted shock system.

Figure 12 compares predicted body force coefficients to balance measurements. Note that to avoid considering effects of base stings, the base pressure drag has been excluded from the balance measurements by employing additional base pressure measurements integrated on the base cross section, and it was also excluded from the integration from CFD. One can see a significant increase in lift and lateral forces as the interbody distance is decreased from $\Delta z / D=3.0$ to 1.06. Drag force (wave drag together with body surface friction drag) is almost constant between $\Delta z / D=1.4-3.0$ and then suddenly increases by a significant margin as the distance between bodies is closed down to $\Delta z / D=1.06$. From the shock wave structures seen in the density gradient contours of Fig. $4 e$ and $6 c$ and plate and body surface streamlines (see Figs. 8 and 11), the difference appears to be due to the shock system existing between the bodies and plate having coalesced into one stronger shock in the region between bodies and plate (similar to so-called 'unstart' phenomena as discussed above), thereby producing an increase in wave drag. Interestingly, the computations tend to predict the experimental lateral and lift forces well while there is a visible overprediction of drag force from $\Delta z / D=1.4$ to 3.0. The magnitudes of difference between computation and experiment are somewhat higher than the indicated experimental error. This difference is potentially due to the level of turbulence closure sophis- 


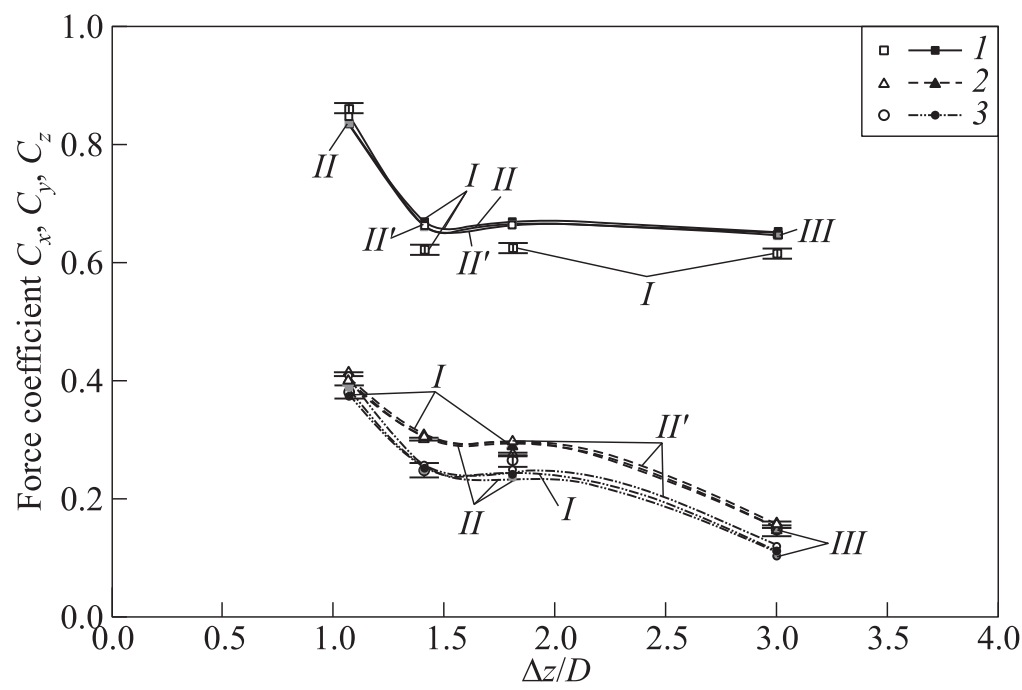

Figure 12 Comparison of predicted (curves with filled symbols) body force coefficients with balance measurements $[2,3]$ (empty symbols) at $\Delta z / D=1.06,1.4,1.8$, and 3.0: $I-56$ million grid cells; $I I-7$ million grid cells, $I I^{\prime}-2$ nd order upwind structured grid JST; $I I I-25$ million cell adapted unstructured JST; $1-C_{x}$ (drag force); $2-C_{y}$ (lift force); and $3-C_{z}$ (lateral force)

tication employed here for predicting surface friction force. Other likely sources of some differences include the flow unsteadiness not captured by steady RANS, impact of using oil-soot mixture to trace streamlines (high viscosity yields zero frequency response to unsteady phenomena) and absence of oil-soot fluid in the computation, chosen method of accounting for sting bases described above. Each of the configurations predicted in general have shown reasonable agreement between numerical simulation and experiment, so it must be acknowledged that the chosen scheme of applying JST with the Spalart-Allmaras turbulence model is quite effective in predicting this sort of complex 3D SWBLI flow. This observation is consistent with prior validation work that was performed [7].

\section{SHOCK CAPTURING OPTIONS}

The study presented herein concentrated on the assumption of steady, fully turbulent flow and predictions mostly employed the JST scheme and SpalartAllmaras turbulence model. This prediction method has been shown to reproduce a range of subsonic and supersonic complex flows well [7] and here the 
correct 3D flow patterns and shock strengths were predicted, although exact shock positions, pressure, and drag magnitudes exhibited some differences from the experimentally determined values.

Considering the results of the present study, it is interesting to look back on earlier work from over two decades ago such as the review of Woodward and Colella [12] who spoke of a set of shock capturing validation studies they considered at the time: "A problem of this nature in two dimensions is presently completely out of the question, as convergence for the most accurate scheme considered here would require a grid of a million zones. The one-dimensional test problem is useful in showing the performance of the schemes under extreme conditions not soon to be encountered in practical two-dimensional calculations." Now, that a solution based on grids containing 1 million - or even 100 million cells - can be achieved, one can evaluate performance of numerical schemes and turbulence models practically in 3D calculations.

For the present calculations, convergence robustness was observed to be significantly higher for the JST scheme compared to the upwind schemes tested. It is worth reviewing some similarities and differences between the schemes. Venkateswaran and Merkle [13] stated: "Artificial dissipation is essential in computational fluid dynamics algorithms in order to eliminate the high wavenumber modes in the solution. Artificial dissipation models may be broadly classified into two families. The first family is associated with central-differenced schemes (Jameson, Schmidt, and Turkel), wherein the dissipation is added as a conscious, explicit step through the introduction of additional higher-order derivative terms. The second family is associated with upwind schemes, where the dissipation is an inherent part of the spatial discretisation." Practical issues in applying such schemes to highly 3D flows such as the one studied herein were pointed out by van Leer [14] where he stated that the Roe scheme should theoretically require only one grid cell across a shock wave to capture it, but that this was only true in practice when the shocks were aligned to the grid: "It can be shown that the upwind flux formula based on Roe's approximate Riemann solver yields a steady normal-shock structure (if aligned with the grid) that contains at most one internal cell. This property is lost for shocks oblique to the grid, which serves as a motivation for the search of truly multi-D upwind methods..." In Fig. 5 discussed earlier in the context of shock interactions arising between the bodies and plate, predictions of shock wave induced density gradient variation are given for both the JST 2nd order central scheme, and the 2nd order upwind schemes of Yee (STVD) and Roe (FDS) - employing two well known flux limiters (van Leer and Roe's superbee). The finest (56 million cell) grid provided a range of 3-6 cells across the shock thickness in this highly $3 \mathrm{D}$ supersonic flow. The next coarsest ( 7 million cell) grid placed 1-3 cells across the same shock thicknesses, so this grid resolution was chosen to compare the two schemes and determine whether the 2nd order upwind schemes would produce similar shock capture to JST on the finest grid. One can see no improvement 
in shock capture by the STVD scheme, and while minor improvements are visible with the Roe scheme employing the superbee limiter, the theoretical shock capture over one cell is not demonstrated when compared to the finest grid JST result. The earlier mentioned drawback of these schemes requiring shocks to be aligned with the grid is thus highlighted. In such a complex flow as the one considered, it is not practical to generate a grid aligned to each of the shocks and, hence, the difference in prediction between the 2nd order upwind schemes and the JST scheme should be expected to reduce compared to what might be seen in a primarily two-dimensional normal shock flow, for instance. Discussing the prediction of shock waves using the JST scheme, Jameson [15] stated: "The JST scheme with scalar diffusive flux captures shock waves with about 3 interior points, and it has been widely used for transonic flow calculations because it is both robust and computationally inexpensive." The present authors' findings have been consistent with this last point, from comparison of computational expense of running JST vs. both the Roe FDS and Yee STVD schemes. Attaining convergence was much more straight forward with JST, and, for instance, the upwind schemes in this study needed to be initiated from a prior solution - making them less desirable in the context of design-cycle analysis. For all schemes tested, shock capture was not adequately demonstrated without a minimum of three grid cells across the shocks (as grid resolution was progressively increased in this study, the predicted shock thicknesses became finer in addition to the eventual increase of number of cells across the shocks). Convergence stability of JST was observed to be significantly closer to monotone than the 2nd order upwind schemes tested. Jameson [15] discussed this problem and pointed to recent efforts to develop practical multidimensional upwind schemes such that "the upwind biasing is determined by properties of the flow rather than the mesh... preliminary results indicate the possibility of achieving high resolution of shocks and contact discontinuities which are not aligned with mesh lines." Thus, both van Leer [14] and Jameson [15] pointed to this need for advanced multidimensional shock capturing schemes, and it appears that the present test case can be used as an example of an application where such schemes would be valuable. Jameson pointed to such work as Paillere and Deconinck [16] and van Leer additionally to these authors highlighted the more recent work of Roe and others on so-called Residual-Distribution Schemes to achieve high order multidimensional shock capturing, stating that the "techniques are starting to look more and more like Discontinuous-Galerkin methods."

In the present study, it has been endeavoured to ensure accurate shock capture by employing very high grid resolution through block-enrichment that was achieved in a manual and iterative process using $\mathrm{F} / \mathrm{T}$, as well as through an automated point-enrichment (or really cell-enrichment) process in $\mathrm{F} / \mathrm{H}$. The next studies should now concentrate on the impact of transition prediction, higher order turbulence closure, and the potential unsteadiness of some configurations. 


\section{CONCLUDING REMARKS}

A set of RANS computations have been carried out for the complex 3D case of two cylindrical bodies in Mach 4 flow, mounted over a flat plate at varying interbody distances. The JST scheme with turbulence closure provided by the Spalart-Allmaras model was employed, and alternative 2nd order upwind schemes were also tested. It was found that when the different spatial discretization schemes were tested on the same grid, affording a range of 1-3 grid cells across the shocks, that there were only slight differences in shock resolution. This was deemed likely to be due to the nonaligned nature of the highly $3 \mathrm{D}$ shock system to the computational grid. Block-enrichment and point-enrichment type approaches were both employed to minimize grid sensitivity of the predictions. The JST scheme and Spalart-Allmaras model produced surface flow separation topology and flowfield structure, static pressure coefficient distributions, and aerodynamic force coefficient predictions that compared well with the experimental values and trends. The large jump in body forces as the interbody distance was decreased from $\Delta z / D=1.4$ to 1.06 was reproduced in the predictions. The chosen numerical scheme and turbulence model were demonstrated to provide acceptable prediction of complex 3D turbulent shock wave/boundary layer interaction. Nevertheless, some differences in predicted positions of separation zones in the vicinity of secondary (reflected) shock waves between the body and plate existed, which will represent the focus of the next stage of research. Grid convergence and shock capturing were shown to be well achieved in the study, and next studies will concentrate on better capturing the turbulence field through the employment of higher order closure, and investigation of potentially laminar/transitional zones, in addition to the potential for unsteadiness.

\section{ACKNOWLEDGMENTS}

The first author would like to thank Ramgen Power Systems for support of the numerical studies. Thanks are also due to Drs. J-C. Bonaccorsi, B. Leonard, and M. Mezine of Numeca for their assistance with the unstructured F/H computations. The second and third authors would like to thank Dr. A. I. Maksimov for his collaboration in the analysis of the experimental data.

\section{REFERENCES}

1. Brodetsky, M. D., E. K. Derunov, A. M. Kharitonov, A. V. Zabrodin, and A. E. Lutsky. 1999. Interference in a supersonic flow over a combination of bodies. 2. The flow about two bodies of revolution over a flat surface. Thermophys. Aeromechanics 6(2):151-57. 
2. Derunov, E.K., A.A. Zheltovodov, and A.I. Maksimov. 2008. Development of three-dimensional turbulent separation in the neighborhood of incident crossing shock waves. Thermophys. Aeromechanics 15(1):29-53.

3. Derunov, E. K., V. F. Volkov, A. A. Zheltovodov, and A. I. Maksimov. 2009. Analysis of supersonic flow around two bodies of revolution near a surface. Thermophys. Aeromechanics 16(1):13-36.

4. Jameson, A., W. Schmidt, and E. Turkel. 1981. Numerical solutions of the Euler equations by finite volume methods using Runge Kutta time stepping schemes. AIAA Paper No. 81-1259.

5. Spalart, P. R., and S. R. Allmaras. 1992. A one-equation turbulence model for aerodynamic flows. AIAA Paper No. 92-0439.

6. Hakimi, N. 1997. Preconditioning methods for time dependent Navier-Stokes equations - application to environmental and low speed flows. Ph.D. Thesis. Vrije Universiteit Brussel, Dept. of Fluid Mechanics.

7. Grosvenor, A.D. 2007. RANS prediction of transonic compressive rotor performance near stall. ASME Paper No. GT2007-27691.

8. Patel, A. 2003. Development of an adaptive RANS solver for unstructured hexahedral meshes. Ph.D. Thesis. Universite Libre de Bruxelles.

9. Delanaye, M., A. Patel, K. Kovalev, B. Leonard, and Ch. Hirsch. 2002. From CAD to adapted solutions for error controlled CFD simulations. RTO-AVT Symposium on Reduction of Military Vehicle Acquisition Time and Cost Through Advanced Modelling and Virtual Product Simulation. RTO-MP-089.

10. Roe, P. L. 1981. Approximate Riemann solvers, parameter vectors, and difference schemes. J. Comput. Phys. 43:357-72.

11. Yee, H. C. 1987. Construction of explicit and implicit symmetric TVD schemes and their applications. J. Comput. Phys. 68:151-79.

12. Woodward, P., and P. Colella. 1984. The numerical simulation of two-dimensional fluid flow with strong shocks. J. Comput. Phys. 54:115-73.

13. Venkateswaran, S., and C. L. Merkle. 1998. Evaluation of artificial dissipation models and their relationship to the accuracy of Euler and Navier-Stokes computations. In: Lecture notes in physics. — Berlin/Heidelberg: Springer. 515:427-32.

14. Van Leer, B. 2006. Upwind and high-resolution methods for compressible flow: From donor cell to residual-distribution schemes. Communications Comput. Phys. $1(2): 192-206$.

15. Jameson, A. J. 2001. A perspective on computational algorithms for aerodynamic analysis and design. Progr. Aerospace Sci. 37:197-243.

16. Paillere, H., and H. Deconinck. 1995. A review of multi-dimensional upwind residual distribution schemes for the Euler equations. In: Computational fluid dynamics review. Eds. M. Hafez and K. Oshima. New York: Wiley. 141-60. 